\title{
DISCRIMINATION OF CHANGES OF LATENCY DURING VOLUNTARY HAND MOVEMENT OF VIRTUAL OBJECTS
}

\author{
Stephen R. Ellis, Mark J. Young*, Bernard D. Adelstein, and Sheryl M. Ehrlich** \\ NASA Ames Research Center \\ Moffett Field, CA
}

\begin{abstract}
Eight subjects' abilities to detect changes in system latency during voluntary lateral hand movement of virtual objects were studied in an immersing virtual environment. A two-alternative forced choice procedure was used in which discrimination of latency was studied with respect to three reference latencies: 27, 94, and 194 msec. Results show that subjects are able to reliably detect changes definitely less than $33 \mathrm{msec}$ and probably less than $16.7 \mathrm{msec}$. Strikingly, for the short latencies we examined, subjects' ability to detect latency changes does not depend upon the base latency we used as a reference. Thus, the discrimination we studied does not appear to follow Weber's law and may provide evidence for quick adaptation to the reference latencies used.
\end{abstract}

\section{INTRODUCTION}

Human interfaces to interactive systems are disturbed by response latency which reduces interactivity, user dexterity, and speed. Because these latencies arise from rendering time, switching delays and transmissions periods, they are likely to persist in systems involving satellite/space communication links even as computing speeds increase. Our previous work has focused on the precision, stability, efficiency and complexity of operator interaction with latency-plagued systems (e.g. Ellis, Bréant, Menges, Jacoby, \& Adelstein, 1997). But there has been relatively little work on users' subjective response to changes in latency which could cue impending degraded performance and also disturb users' sense of immersion in "virtual" tasks (see Uno \& Slater, 1997; Welch, Blackmon, Liu, Mellers, \& Stark, 1996). In particular, operators' psychophysical functions describing sensitivity to detection of the visual consequences of latency change have not been measured. We have measured these functions for the first time for hand movement of nearby virtual objects.

The literature on manual control has long established that latency in displays or controls has a major negative impact on performance (Smith and Smith, 1962; Ferrell, 1965; Sheridan, 1992). In general, the effect involves a reduction in control accuracy which ultimately drives the operator to adopt a "move and wait" strategy when latency exceeds about $300 \mathrm{msec}$. Operator compensation for a delay usually requires the ability to predict the future state of a tracked element.

\footnotetext{
* Gestalt Technologies, Mountain View CA

** Interval Research Corporation, Palo Alto, CA
}

Display delays have also been shown to interfere with operator adaptation to other display imperfections such as static positional distortions in vision (e.g. Held, Efstathiou, \& Greene, 1966). Consequently, but not surprisingly, delays in visual displays have been shown to have major impact on overall operator productivity in the workplace (Doherty \& Thadhani, 1982).

Interest has more recently moved away from the performance impact of delay and update rate onto their subjective impact. These two factors in particular have been studied in virtual environment simulations. In these studies delay and update rate have been considered as factors affecting the operators' sense of presence in the environment. (Sheridan, 1992; Barfield \& Hendrix, 1995; Welch et al., 1996; Ellis, Dorighi, Bréant, Menges, Adelstein \& Jacoby, 1997). These dynamic aspects of displays are particularly potent influences on users' sense of presence because they tend to swamp other factors influencing important virtual environment parameters such as positional fidelity and dynamic registration error (Holloway, 1997).

Since transmission delay is inescapable in many virtual environment, teleoperation, or augmented reality applications, interest naturally is directed to how detectable differing levels of delay might be. Poulton (1974, p. 202) reports that manual tracking performance is reliably reduced with delays as low as 40 msec because such delay may not be "appreciated" by the operator. But his book contains no specific reports of the differential discriminability of various levels of delay. In fact, we have not been able to find such data in the literature.

Accordingly, the following experiment has been designed to provide the first measures of human op- 
erators' discrimination of the consequences of latency during hand movement within an immersing virtual environment. Subjects' psychophysical functions for the discrimination of latency were measured with a two alternative forced choice technique. Since the subjects were asked to only make a single stereotyped hand movement, strictly speaking they are not discriminating latency, but only its visual consequences. These appear as uncommanded movements of virtual objects that are observed as is illustrated in Figure 1 schematically showing a user of a virtual environment beginning to move a virtual object "attached" to an unseen hand. As the user's hand moves to the right, the virtual object lags behind due to the system lag and a spatial registration error develops in the opposite direction of the movement.

\section{Simulation}

\section{METHODS}

The environmental simulation used for the experiments was produced on a SGI ONYX graphics computer with RE-2 graphics viewed through a Virtual Research V8 head-mounted display. FasTrak hand and head position sensors were used with a custom dual-serial driver which has achieved dynamic performance comparable to a previously described parallel driver (Jacoby, Adelstein, \& Ellis 1996). Because we use two Polhemus sources simultaneously, we are able to load head and hand position and orientation into shared memory at $120 \mathrm{~Hz}$. Notably for the simulation content that we used, the system has been able to maintain a regular $60 \mathrm{~Hz}$ simulation update rate for a stereoscopic display. Minimum full system latency has been measured to be $27 \pm 5 \mathrm{msec}$. Since the measurement technique we use contributes to some measurement variability, the actual latency variation is $<5$ msec. The unique dynamic performance of our system make the following experiment possible.

\section{Task}

An immersing virtual environment simulation was used to present subjects with a simple virtual environment giving the impression that they are looking at a multifaceted, neutrally colored, $10 \mathrm{~cm}$ diameter faceted virtual ball located at arm's length. The ball is lit by two virtual light sources, one ambient and one directed so as to make the facets visible and to appear somewhat like a faceted 3/4 moon. Maximum luminance as seen by the subject was midphotopic, about $50 \mathrm{~cd} / \mathrm{m}^{2}$. No other environmental elements are simulated.

Subjects seated within $60 \mathrm{~cm}$ of the FasTrak transmitters are asked to move the ball back and forth once through an arc subtending 48 degrees of visual angle, the full binocular field of view of the head mounted display used. In this case the virtual ball was electronically "attached" to the subjects' dominant hand so it appeared to follow it as it was moved. Since the subjects' hand was not visible in the virtual environment, any system latency in the rendering of the virtual ball appeared as a transient mismatch in the spatial position of the virtual ball and felt position of the hand.

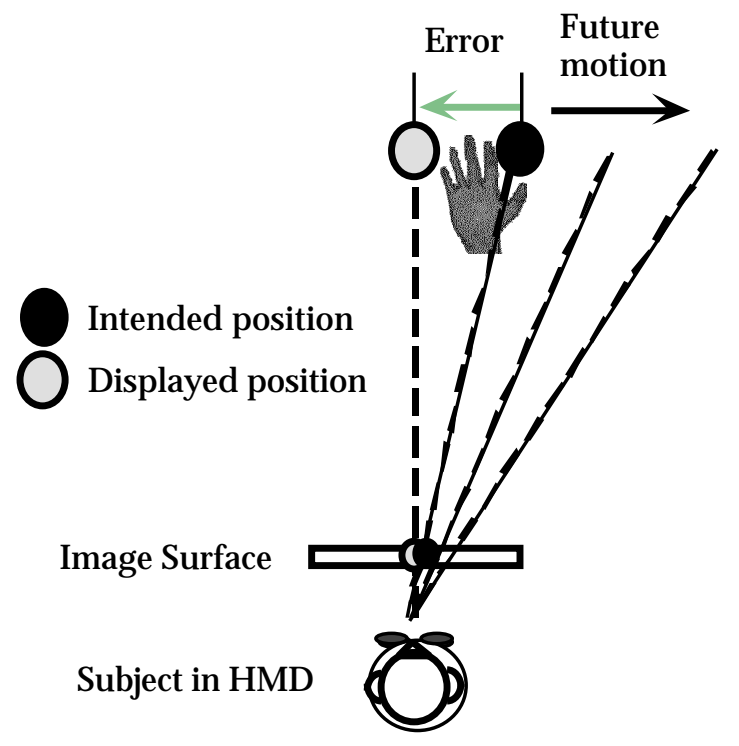

Figure 1. Subject moves a virtual object in an immersing virtual environment presented via a head mounted display. Because of the occluding display each subject's actual hand was invisible to the subject during the movement.

Initial adjustment of the virtual environment was made to make the subject's hand and ball appear statically to be approximately coincident. Subjects were instructed to move the virtual ball at arm's length back and forth through a distance subtending the full 48 degree field of view of the head mounted display. This movement was paced by clicks from a metronome set at 72 counts/minute $(\sim 0.4 \mathrm{~Hz})$.

In the actual experiment subjects were presented with blocks of comparisons derived from one of three reference latencies. Each block would be based on four possible subblocks which were randomly ordered. Subjects were presented the base latency vs. the base latency, the base vs. the base + a number of increments (16.7 msec/increment), the base + increments vs. the base, or base + increments vs. base + increments. The first and last group were catch trials, i.e. compare conditions with equal latency. The use of either an increase or decrease in latency was intended to simulate a realistic graphics environment in which latency may either increase or decrease. Subjects were required to indicate whether the two conditions were the same or different by 
button presses on a hand-held response device. A trigger on it advanced to the next condition.

For each of the seven latency comparisons use for all subjects the detection probabilities and false alarms reported below were based on 64 trials in which $25 \%$ presented a difference and $75 \%$ were catch trails. This distribution of trials was based on pilot studies which indicated that at 50:50 distribution would not produce a sufficient number of false alarms to be able to determine if their rate held constant when experimental variables were changed. One to seven $16.7 \mathrm{msec}$. steps of latency were randomly presented for 27, 94, and $194 \mathrm{msec}$ base latencies. These references were randomly either incremented or decremented by the step for each comparison. All tests were blocked by base latency and increments. Blocks were independently randomized per subject.

\section{Subjects}

Complete data for eight subjects are presented below. All subjects were trained to the discrimination task by at least about 1 hour of familiarization and practice before any data were collected. During this time, the subject experienced a variety of the experimental conditions to become familiar with the experimental variation. Subjects were laboratory personnel and cooperative friends familiar with the task and who were able to tolerate the approximately one day of data collection required during which high vigilance had to be maintained. Subjects were blind to the specific experimental conditions during each judgment. Breaks were allowed after every 20-30 minutes of data collection to maintain concentration and allow subjects to rest their dominant arm with which they "held" the virtual object. All subjects have been right handed in an age range of 20 to 51 .

\section{RESULTS}

As shown by the across subjects means plotted in the left panel of Figure 2, the psychophysical functions for the three different reference latencies substantially overlap, all being within the range of uncertainty delimited by the standard error bars. Similarly, the false alarm rates overlap and remain approximately constant for all three reference latencies. (Corresponding results are also evident in plots of medians and semi-interquartile range.).

\section{DISCUSSION}

The most striking feature of the measured psychophysical functions is that neither the correct detections of latency differences (Hits) nor the false detections of difference (False Alarms) are affected by the differing base latencies. Because of the flat False Alarms functions, observers' judgment criteria also appears to be fairly constant for the different conditions.

\section{Hits}

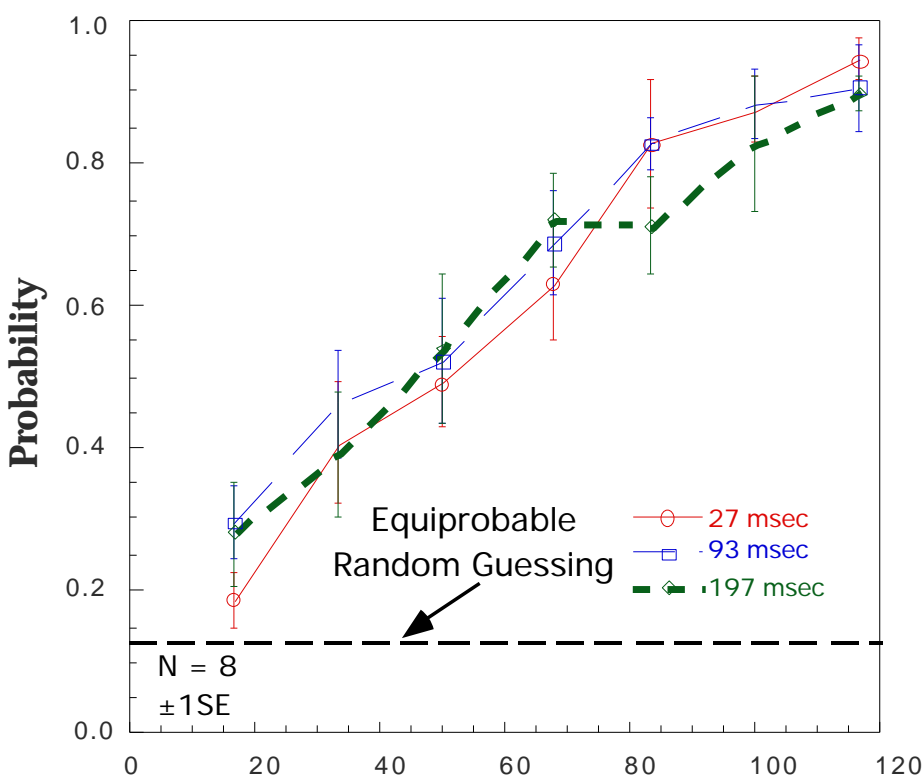

\section{False Alarms}

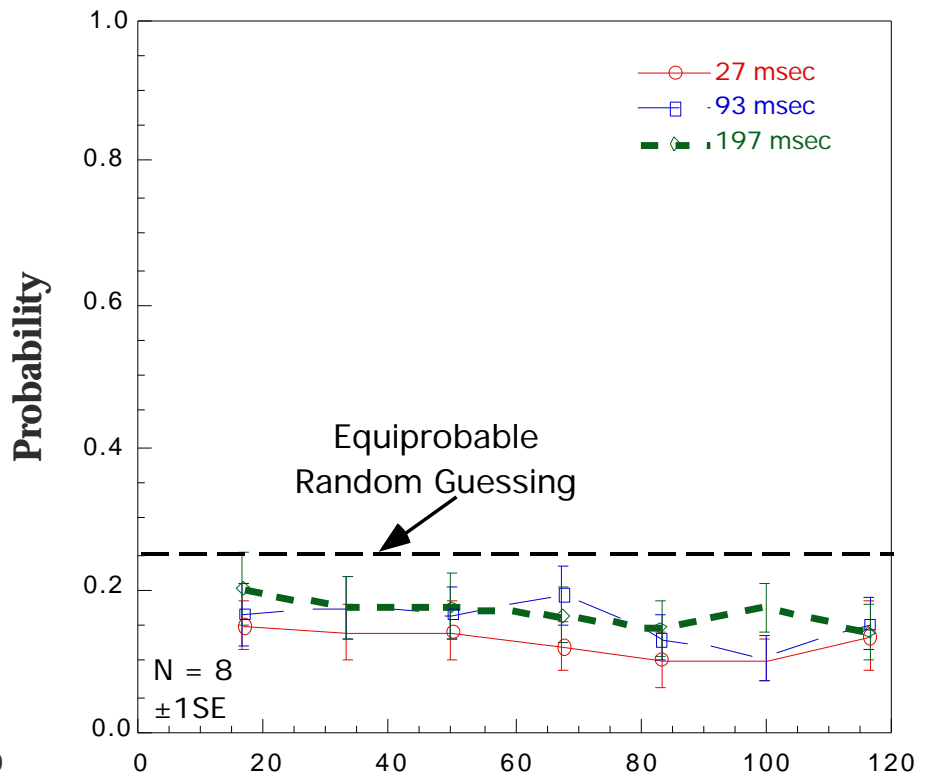

Figure 2. Hit probability or rate is that of a correct detection. False alarm probability or ate is that of an incorrect claim of detecting a difference in latency. 
All eight subjects show this behavior individually. The detection threshold based on a 75\% correct criteria, or any criteria for that matter, is essentially the same for the three base latencies even though the bases differ by a factor of approximately seven.

The discrimination, in fact, does not seem to follow Weber's Law. If Weber's Law were in effect, the threshold would be a fixed proportion of the base latency, and one would accordingly expect the threshold for the discrimination with respect to the shortest base latency to be reached before those for the longer base latencies.

In order to get a sense of observer general sensitivity to detecting latency it is interesting to consider possible results from some simple random response strategies that might be adopted by subjects. Consider for example a subject who simply guessed at random that trials with differences in latency and those with no difference occurred equally often. Since it is reasonable to assume subjects' guesses would be independent of the randomized experimental conditions, the random guessing strategy leads to a hit expectation of $(0.25)(0.5)=0.125$ and a false alarm rate of $(0.75)(0.5)=0.375$. These values are plotted as lines on Figure 2 for reference.

Alternatively, if the subjects had been randomly guessing but matching the presentation probabilities, the expected hit probability would be

$(0.25)(0.25)=0.062$ and the false alarm rate would be $(0.75)(0.75)=0.562$. Clearly, the subjects appear to be performing in aggregate well above chance as modeled by these alternative assumptions.

Interestingly subject-by-subject analysis of each individual subject's results show that for six of the eight subjects, hit rates for the $16.7 \mathrm{msec}$ latency difference exceeded that corresponding to the equally likely random guessing strategy. All subjects' results exceeded this expectation for latency differences of 33 msec or above (sign test: $p<.01$ ). Subjects' individual hit rates universally exceed those expected by the probability matching hypothesis (sign test: $\mathrm{p}<.01$ ). Thus, though a more statistically powerful test might show one step of $16.7 \mathrm{msec}$ latency change discriminable, we may conclude from existing data that two steps are definitely discriminable.

It should be noted that the stereotyped hand movements used preclude interpretation that the subjects are detecting changes of latency per se. Subjects may only be responding to the magnitude of a felt visual displacement of the virtual object with respect to their hand. This displacement would be most noticeable during the beginning or end of a lateral hand movement at which time the mismatch in position and direction sense between propioception and vision would be greatest. Figure 3 illustrates some of the specific features of velocity and position that would allow latency to be indirectly discriminated.
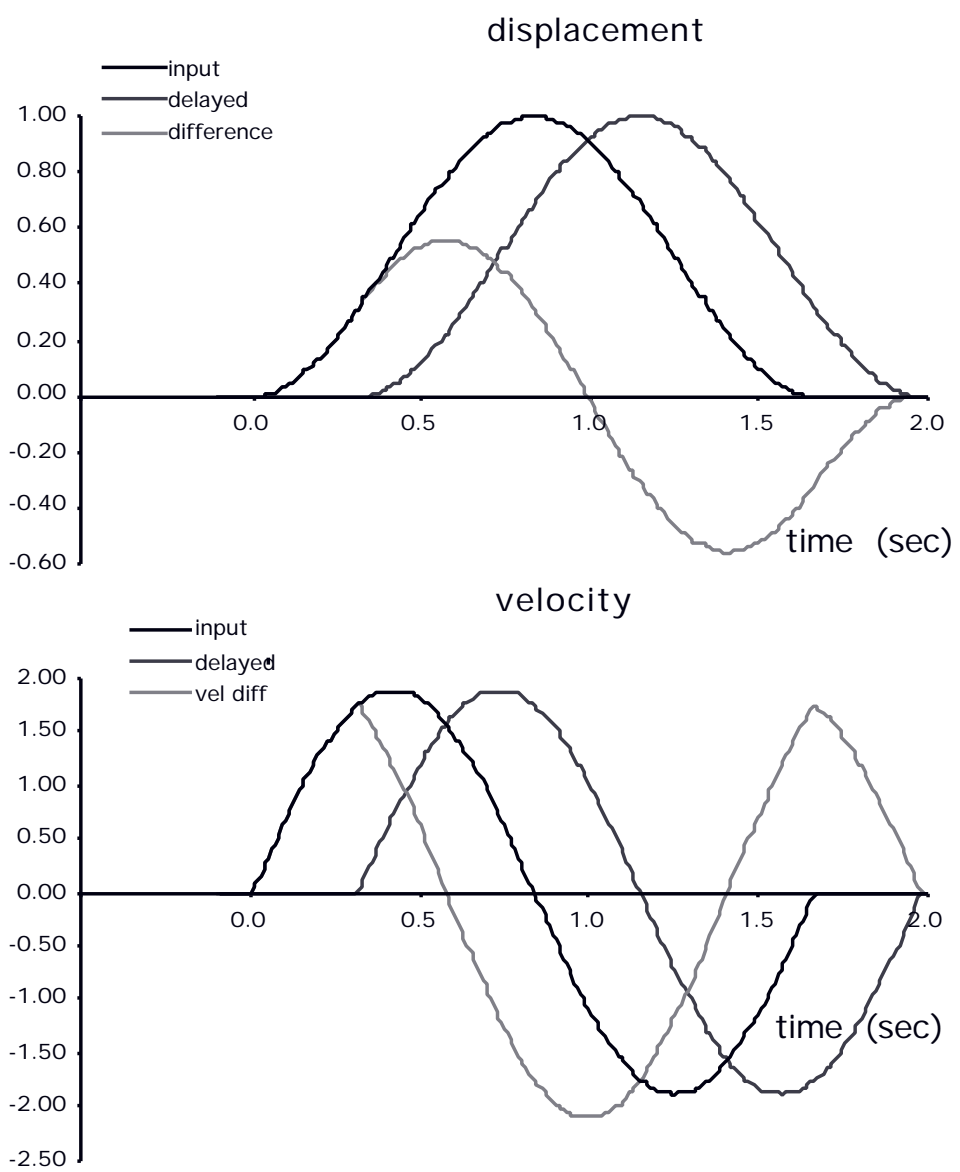

Figure 3. Input and error in arbitrary units produced during a half cycle of pendular movement of a virtual object positioned by a user's hand with $313 \mathrm{msec}$ system latency.

A detection of latency based on perceived spatial offset of attached virtual objects during hand movement requires that the observer be able to take their speed of movement into account. The would need to discriminate ratios of felt visual displacement to perceived velocity not just displacement alone. Future investigations will examine whether latency itself may be discriminated by forcing subjects always to make movements of different speeds when comparing intervals with potentially different latencies. It should however remain noted that since the speed of hand movement of virtual objects will over short periods of time typically remain more or less constant. It is interesting to consider what features of the stimulus could be used to distinguish the amount of latency in presentation of the virtual object during the half cycle of movement generated by the subjects. Assuming 
felt arm position as the input reference as in Figure 3, they could for example be attending to RMS error between the felt position of the object based on their hand position and the judged visual position of the virtual object. Alternatively, they could be attending to the maximum error between visual and felt position. Similarly, they could be attending to integrated errors in proprioceptively sensed velocity versus visually sensed velocity or to maximum velocity error.

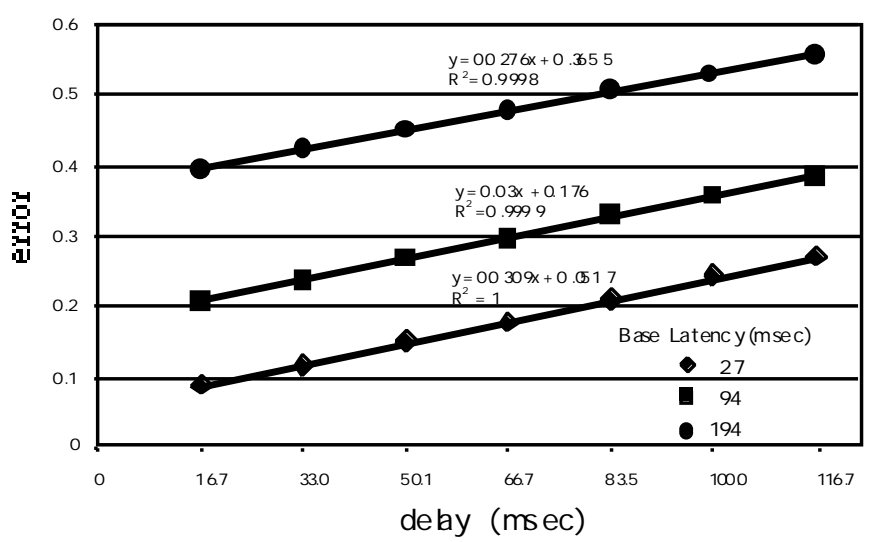

Figure 4. Change in peak position error

Some insight into how specific indicators of dynamic position error might reveal system latency can be found in Figure 4. It plots an estimate of the peak position error that would have been produced during the pendular lateral movement illustrated in Figure 3. This figure shows how the peak position error would change for of up to seven steps of latency change at each of the different base latencies.

From this figure it is apparent that increases in peak error as latency changes are introduced for each different base at approximately the same rate; i.e. all curves have approximately the same slope. Thus, if the discriminability of latency change were based on the increase of this specific indicator and not its absolute value, one could expect that the discrimination results plotted in Figure 2 should not depend upon the base latencies. This kind of response, which in fact was observed, would occur if the subjects were very quickly to adapt to the base latency level, treating it as a reference for subsequent change. Since for all the psychophysical judgments, the different base latencies were presented in blocks, future analysis of the data may be able to determine if an adaptation to each change of the base latency occurs.

The essentially identical psychophysical functions for the different base latencies means that users of long latency virtual environment systems will be as sensitive to changes in latency as those who use prompter, more advanced systems. Designers will not be able to count on long latency interactivity to "smear over" variations in latency of the size we have examined. Furthermore, the data collected so far suggest that designers should expect users to in general be able to notice changes in latency when the change is above about 33 msec.

\section{REFERENCES}

Barfield, W., and Hendrix, C., The Effect of Update Rate on the Sense of Presence within Virtual Environments, Virtual Reality: Research, Development, and Application, Vol. 1, (No. 1), 3-15, 1995.

Barfield, W., Hendrix, C., Bjorneseth, O., Kaczerak, K., and Lotens, W., Comparison of Human Sensory Capabilities with Technical Specifications for Virtual Environment Equipment, Presence, 4, 329-356, 1995.

Doherty, W. J. \& Thadhani, A. J. (1982) The economic value of rapid response time. IBM report GE20-0752-0 (11/82).

Ellis, Stephen R., Bréant, François, Menges, Brian M, Jacoby, Richard H. and Adelstein, Bernard D. (1997) Operator interaction with virtual objects: effects of system latency. Proceedings of HCI'97 International. San Francisco, CA. pp. 973-976.

Ellis, Stephen R., Dorighi, Nancy. S., Menges, Brian. M., Adelstein, B. D. and Jacoby, Richard. H. (1997) In search of equivalence classes in subjective scales of reality. Proceedings of HCI'97 International. San Francisco, CA. pp. 873-876.

Ferrell, W.R. (1965) Remote manipulation with transmission delay, IEEE Trans. on Human Factors in Electronics, HFES 6, no, 1.

Held, R., Efstathiou, A., and Greene, (1966). Adaptation to displaced and delayed visual feedback from the hand, Journal of Experimental Psychology, 72, 887-891.

Holloway, R. L. (1997) Registration error analysis for augmented reality. Presence, 6, 4, 413-432.

Jacoby, R., Adelstein, B.D. \& Ellis, S.R. (1996) Improved temporal response in virtual environment hardware and software. IS\&T/SPIE Proceedings, Conference. 2653B, Session 2653-39, pp. 271-284.

Uno, S. \& Slater, M. (1997) The sensitivity of presence to collision response. IEEE VRAIS Proceedings, pp. 95-101.

Poulton, E.C. (1974) Tracking skill and manual control. Academic: New York.

Sheridan, T.B. (1992) Musings on telepresence and virtual presence. Presence, 1(1), 120-125, pp. 212-227.

Smith, K.U., \& Smith, W. M. ( 1962) Perception and action, Saunders, Philadelphia, pp. 247-277.

Welch, R. B., Blackmon, T.T., Liu, A., Mellers, B. A., and Stark, L. W. (1996) The effects of pictorial realism, delay of visual feedback, and observer interactivity on the subjective sense of presence. Presence, 5, 3, 263-273

Acknowledgments: Research support: NASA RTOP 131-2030-51-12, Thanks to Eric Barrett, for programming the data collection software and to Matthew Panos-Ellis and Darren Heinrichsen for pilot testing and data collection. 